Relations industrielles

Industrial Relations

\title{
Industrial Relations in Canada. by Stuart Jamieson, Toronto, The Macmillan Company of Canada, 1957, pp. 144.
}

\section{Gérard Dion}

Volume 13, numéro 1, janvier 1958

URI : https://id.erudit.org/iderudit/1022479ar

DOI : https://doi.org/10.7202/1022479ar

Aller au sommaire du numéro

Éditeur(s)

Département des relations industrielles de l’Université Laval

ISSN

0034-379X (imprimé)

1703-8138 (numérique)

Découvrir la revue

Citer ce compte rendu

Dion, G. (1958). Compte rendu de [Industrial Relations in Canada. by Stuart

Jamieson, Toronto, The Macmillan Company of Canada, 1957, pp. 144.]

Relations industrielles / Industrial Relations, 13(1), 110-111.

https://doi.org/10.7202/1022479ar

Tous droits réservés (C Département des relations industrielles de l’Université Laval, 1958
Ce document est protégé par la loi sur le droit d'auteur. L’utilisation des services d'Érudit (y compris la reproduction) est assujettie à sa politique d'utilisation que vous pouvez consulter en ligne.

https://apropos.erudit.org/fr/usagers/politique-dutilisation/ 
There is an urgent need for a study of human relationships in industry and elsewhere and we look forward to more work from this author and his associates.

\section{W. Harding le Riche, M.D. University of Toronto.}

\section{Industrial Relations in Canada by} Stuart Jamieson, Toronto, The Macmillan Company of Canada, 1957, pp. 144.

Cet ouvrage est le premier d'une série de publications consacrées aux nrouvements ouvriers et aux relations industrielles en dehors des Etats-Unis. Il est divisé en quatre parties.

La première décrit le contexte économique et social dans lequel le syndicalisme et la pratique de la négociation collective se sont développés au Canada. L'auteur fait toujours référence à la situation américaine. Il explique la lenteur de la croissance du mouvement syndical et les raisons pour lesquelles les ouvriers canadiens sont groupés dans des unions américaines.

Le second chapitre est consacré spéoifiquement au syndicalisme ouvrier dont il retrace les grandes lignes de l'histoire. Influencé au début par la Grande-Bretagne dont le Canada est colonie, le syndicalisme canadien, à partir de la deuxième moitié du siècle derrier, est surtout une extension du syndicalisme américain auquel il emprunte ses politiques d'organisation et son idéologie et dont il est partie intégrante par l'affiliation. Sans cet apport américain en hommes, en ressources et en expérience, le syndicalisme canadien ne se serait pas répandu aussi rapidement. Par contre, l'influence américaine a contribué à donner naissance à des sursauts périodiques de protestations qui se sont exprimés par la création de groupements purement canadiens. C'est dans le Québec que cette tendance a été la plus forte à cause de l'existence d'une culture et d'une religion différentes. Mais ce phénomène n'est pas exclusif à cette province. Historiquement, une des caractéristiques du mouvement ouvrier canadien, si on le compare à celui des Etats-Unis, et à celui de la Grande-Bretagne, c'est qu'il a été, au cours de sa brève existence, marqué par de nombreuses divisions internes et beaucoup de conflits. Mais aujourd'hui l'unité est presque réalisée puisqu'il n'existe que deux centrales, le Congrès du Travail du Canada et la Confédération des Travailleurs Catholiques du Canada et que même cette dernière a accepté le principe d'une affiliation.

Le troisième chapitre étudie la structure, le gouvernement et les politiques des syndicats ouvriers canadiens. L'auteur développe longuement le genre de rapports qui existent entre les syndicats canadiens et les syndicats américains. Il discute une série d'hypothèses tendant à expliquer ce phénomène unique au monde d'un syndicalisme relevant d'un pays étranger et contrôlé par des étrangers. Il parle de la crainte de la concurrence tant sur le plan de la main-d'oeuvre que du produit, des avantages pécuniers que pourraient retirer les unions américaines, de la nécessité d'expansion pour survivre et de croître. Mais d'après lui, les raisons principales se trouvent dans le fait que ce sont les Canadiens qui désirent qu'il en soit ainsi parce qu'ils en retirent de grands avantages. De plus, les similitudes économiques et culturelles entre le Canada et les Etats-Unis sont telles que, nulle part au monde, on rencontre pareille situation. Aussi les travailleurs canadiens, comme les professionnels et les hommes d'affaires, les universitaires, trouvent dans des groupements américains un moyen pour satisfaire à leurs aspirations et à leurs besoins qui est assez efficace pour compenser les inconvénients d'une «domination» réelle ou imaginaire. Ici, l'auteur explique dans les détails le genre de rapports qui existe entre les groupements locaux ou régionaux canadiens et les unions américaines. Il montre le degré d'autonomie que possèdent les groupements canadiens, selon qu'il s'agit d'unités autrefois affiliés à la FAT ou au COI. Puis il établit un parallèle entre le CTC et l'AFL-CIO en regard de l'action politique.

Le dernier chapitre porte surtout sur le cadre juridique dans lequel évoluent les relations industrielles au Canada et sur l'attitude des gouvernements par rapport au syndicalisme et à la négociation collective.

L'ouvrage se clôt par une liste, assez incomplète, d'ouvrages dont il suggère la lecture. 
L'étude du professeur Jamieson est d'une lecture facile. Elle a le mérite de condenser dans un nombre restreint de pages les éléments essentiels à la compréhension du mouvement ouvrier canadien. Il s'est glissé, ici et là, quelques erreurs de détail; certaines hypothèses mériteraient d'être approfondies davantage. Mais dans l'ensemble le lecteur y trouvera sûrement le meilleur essai de synthèse publié à date sur un sujet très complexe, le mouvement ouvrier canadien. L'auteur l'a bien situé dans son contexte géographique, social, économique, juridique et politique.

\section{GÉrard Dion}

\section{Mgr Guerry, La Doctrine sociale de} l'Eglise, 1 vol., 191 pp. Bonne Presse. Paris, 1957.

Les ouvrages sur la doctrine sociale de l'Eglise ne manquent pas. Plusieurs ont vu le jour en ces dernières années. Certains ont des qualités incontestées. Tels ceux du R.P. Vilain, s.j., et du R.P. Van Gester, o.p. D'autres pour diverses raisons sont moins recommandables.

Voici qu'un évêque français, bien connu en notre milieu pour ses travaux sur l'Action catholique, vient de publier en un petit volume une lettre pastorale au clergé et aux militants de son diocèse intitulée La Doctrine sociale de l'Eglise, son actualité, ses dimensions. son rayonnement.

Cet ouvrage est un des meilleurs que nous connaissons. On n'y trouve pas de «vasage », ni de jérémiades, ni de sentimentalisme. Son grand mérite est d'exprimer l'essentiel avec précision et concision. Grâce à des définitions claires et à des distinctions lumineuses, il dissipe les confusions entretenues par ceux qui, malgré leurs bonnes intentions, n'ont pas renouvelé leur vocabulaire ni assimilé les développements de l'enseignement social de l'Eglise depuis vingtcinq ans. L'auteur ne nous écrase pas non plus sous des montagnes de citations pour mieux cacher sa pensée, bien qu'il donne toujours en note les références les plus importantes.

L'ouvrage comprend d'abord une substantielle introduction où est dit en quoi consiste la doctrine sociale de
l'Eglise, pourquoi l'Eglise a une doctrine sociale, et où est fait réponse à un certain nombre d'objections. La première partie est consacrée à la conception de l'homme apportée au monde par cette doctrine. La seconde a trait à la conception de l'économie sociale. Mgr Guerry se propose de compléter son étude dans une autre étude où il exposera la conception chrétienne de la communauté humaine.

Cet ouvrage se recommande sans réserve à cause de l'autorité de son auteur et surtout à cause de ses qualités intrinsèques. Il peut fort bien être utilisé comme manuel pour un cours sur la doctrine sociale de l'Eglise. On le distribue aux Presses Universitaires Laval, Québec.

GÉrard Dion

Code du travail - Labor Code, par Gérard Picard, Montréal 1957. 1 volume bilingue, 245 pp.

Le président général de la CTCC vient de publier sous ce titre un essai de codification de la législation du travail pour la province de Québec. Il y exprime sa propre conception de la législation du travail ainsi que ce qu'elle doit, selon lui, comprendre. Il le fait en tenant compte des mesures législatives existantes et surtout des pratiques courantes en notre pays et de sa longue expérience dans lorganisation syndicale et la négociation de conventions collectives. Voilà pourquoi dans son essai on trouvera plusieurs suggestions que l'auteur croit devoir offrir. Comme il serait trop long de faire une étude critique de chacune des innovations, il vaut mieux se contenter de les présenter aux lecteurs, quitte à revenir plus tard sur l'une ou l'autre des originalités de ce projet. Les voici.

Le Code du Travail érige la législation du travail en un système juridique particulier et remplace les lois éparses et fragmentaires édictées depuis environ un demi-siècle.

Le Code du Travail prévoit qu'il doit y avoir consultation officielle des associations patronales et des organisations syndicales de travailleurs avant la formation de tout organisme consultatif, administratif ou semi-judiciaire relevant de la juridiction du Ministre du Travail. 